\title{
The Latin American Association of Cardiac and Endovascular Surgery statement regarding the recently released American Heart Association/American College of Cardiology Guideline for the Management of Patients With Valvular Heart Disease 2020
}

Victor Dayan, MD, PhD, ${ }^{\mathrm{a}}$ Ovidio A. Garcia-Villarreal, MD, ${ }^{\mathrm{b}}$ Alejandro Escobar, MD, ${ }^{\mathrm{c}}$ Javier Ferrari, MD, ${ }^{\mathrm{d}}$ Eduard Quintana, $\mathrm{MD}, \mathrm{PhD},{ }^{\mathrm{e}}$ Mateo Marin-Cuartas, $\mathrm{MD}, \mathrm{PhD},{ }^{\mathrm{f}, \mathrm{g}}$ and Rui Almeida, MD, MSc, $\mathrm{PhD}{ }^{\mathrm{h}}$

Scientific evidence in the cardiologic arena has progressed enormously in the last couple of years. It is a huge challenge for respected societies such as the American Heart Association (AHA) and American College of Cardiology (ACC) to undertake the burden of providing to the world their recommendations for clinical practice based on this evidence. The Latin American Association of Cardiac and Endovascular Surgery (LACES) thanks the authors involved in such a task.

For a growing association that represents an economic and healthcare reality that is different from others, we have decided to carefully select guidelines that consider our socioeconomic situation. As such, in this statement, we will highlight the aspects of the recently released AHA/ACC Guideline for the Management of Patients With Valvular Heart Disease 2020 with which we disagree, intending to support Latin American surgeons in their practice.

From the ${ }^{a}$ Centro Cardiovascular Universitario, Montevideo, Uruguay; ${ }^{b}$ Mexican College of Cardiovascular and Thoracic Surgery, Mexico City, Mexico; ${ }^{c}$ Universidad CES, Medellin, Colombia; ${ }^{\mathrm{d}}$ Colegio Argentino de Cirujanos Cardiovasculares, Buenos Aires, Argentina; ${ }^{\mathrm{e}}$ Cardiovascular Surgery Department, Hospital Clinic Barcelona, Barcelona, Spain; ${ }^{\mathrm{f}}$ University Department of Cardiac Surgery, Leipzig Heart Center, Leipzig, Germany; ${ }^{\mathrm{g}}$ Department of Cardiothoracic Surgery, Stanford University, Stanford, Calif; and hACES, University Center Assis Gurgacz, Cascabel, Brazil

Disclosures: The authors reported no conflicts of interest.

The Journal policy requires editors and reviewers to disclose conflicts of interest and to decline handling or reviewing manuscripts for which they may have a conflict of interest. The editors and reviewers of this article have no conflicts of interest.

Received for publication Jan 21, 2021; revisions received Jan 21, 2021; accepted for publication Jan 21, 2021; available ahead of print Feb 26, 2021.

Address for reprints: Victor Dayan, MD, PhD, Hospital de Clinicas, Cirugía Cardiaca, 26 de Marzo 3459, Ap 602, Montevideo 11300, Uruguay (E-mail: victor_dayan@ hotmail.com).

J Thorac Cardiovasc Surg 2021;162:584-6

0022-5223

Copyright (c) 2021 Published by Elsevier Inc. on behalf of The American Association for Thoracic Surgery. This is an open access article under the CC BY license (http:// creativecommons.org/licenses/by/4.0/).

https://doi.org/10.1016/j.jtcvs.2021.01.139

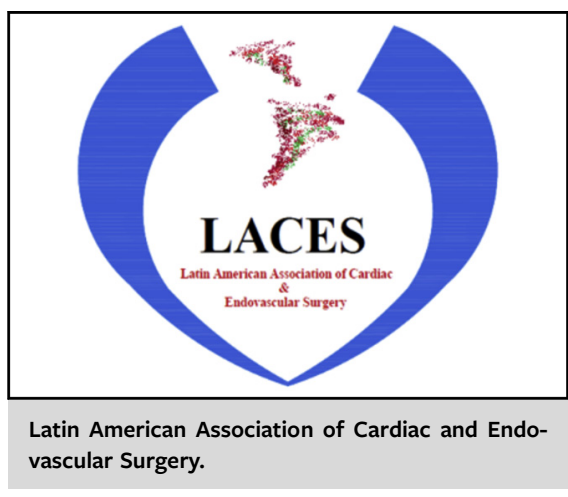

\begin{abstract}
CENTRAL MESSAGE
Guidelines on the management of cardiovascular disease are constructed on the basis of the best clinical evidence. We believe the recently released $\mathrm{AHA} / \mathrm{ACC}$ Guideline for the Management of Patients With Valvular Heart Disease 2020 have important sections that fail on this major premise; therefore, our association will not support them.
\end{abstract}

See Commentaries on pages 587 and 588.

\section{AORTIC STENOSIS}

Trials on transcatheter aortic valve implantation (TAVI) and surgical aortic valve replacement (SAVR) have been constructed on the basis of surgical risk. For this reason, the previous AHA/ACC guidelines for the management of patients with valvular heart disease based their recommendation for the type of intervention on surgical risk. Although we support the concept that age, expected survival, and valve durability are the cornerstones for the patient and heart team discussion, trials have not evaluated 
outcomes on the basis of age. Furthermore, the age range used to support TAVI is well below the mean age of the low-risk trials (73 year old for PARTNER 3 and 74 year old for EVOLUT Low Risk), and there is absolutely no reference to support this range defined by the authors. Therefore, LACES considers it an important methodological flaw subject to high risk of reversal to recommend as Class of Recommendation (COR) I Level of Evidence A (the highest imprimatur of guideline recommendations) any indication for TAVI or SAVR based on age. We consider this discrepancy of utmost importance because the AHA/ ACC recommendations will guide the treatment and provide the legal framework in several countries with thousands of patients, which in this case are devoid of scientific evidence.

LACES does not support any COR I level of evidence A recommendation that is not supported by large randomized control trials. Large randomized control trials have been constructed on the basis of surgical risk. Therefore, we do not support any recommendation on TAVI or SAVR based on age.

The authors have clearly stated the importance of life expectancy and valve durability to help decide the best strategy. Nonetheless, there is no mention about the long-term risk of paravalvular leak or permanent pacemaker implantation on long-term survival in low-risk and young patients. The PARTNER 2 trial has shown patients with a mild paravalvular leak to have worse survival at 5 years $(P=.06)$ than patients with no or trace paravalvular leak. ${ }^{2}$ We believe this issue to be as important as valve durability and therefore should be seriously incorporated in the decision for patients with more than 5 years of life expectancy. Until there are no data on its detrimental effect, we do not believe it is safe to recommend TAVI in patients with more than 5 years of life expectancy.

Because there is no evidence for more than a median of 5 years of follow-up, to support the safety of TAVI in intermediate- and low-risk patients, and regarding the detrimental effect of paravalvular leak, LACES does not support any COR I for TAVI in patients with a life expectancy more than 5 years.

Surgical risk defined by the current guidelines is another novel topic in which this association has a different view. High risk has been defined by the current guidelines as any of the following: Society of Thoracic Surgeons risk greater than $8 \%, 2$ or more indices in frailty, 1 to 2 organ system compromise not to be improved postoperatively, and possible procedure-specific impediment.

Organ system compromise has been defined as cardiac dysfunction (severe left ventricle [LV] systolic or diastolic dysfunction or right ventricle dysfunction, fixed pulmonary hypertension); kidney dysfunction (chronic kidney disease, stage $\leq 3$ ); pulmonary dysfunction (forced expiratory volume in 1 second $<50 \%$ or diffusing capacity for carbon monoxide $<50 \%$ of predicted); central nervous system dysfunction (dementia, Alzheimer's disease, Parkinson's disease, cerebrovascular accident with persistent physical limitation); gastrointestinal dysfunction (Crohn's disease, ulcerative colitis, nutritional impairment, or serum albumin $<3.0 \mathrm{~g} / \mathrm{dL}$ ); cancer (active malignancy); and liver dysfunction (any history of cirrhosis, variceal bleeding, or elevated international normalized ratio in the absence of vitamin $\mathrm{K}$ antagonist therapy).

There is no reference to support defining high surgical risk under these conditions, and we consider the organ system compromise definition to be very broad, resulting in a high percentage of patients in this category who will receive a treatment for which there is no evidence to support superiority.

LACES considers that surgical risk stratification should continue to be based on validated scores that result from complex statistical methods and therefore do not support defining high surgical risk based on criteria that are not derived from large data-adjusted survival analyses.

Current guidelines have excluded completely the option of SAVR in patients at high surgical risk. The authors have provided similar recommendations for high surgical risk and prohibitive surgical risk. This recommendation is unacceptable, and our association will not support or endorse it for the following reasons. Trials have separately evaluated each of these surgical risks (PARTNER 1A and 1B), providing strong and solid evidence based on the population of patients included: PARTNER 1A compared SAVR with TAVR in patients at high risk, and PARTNER 1B compared medical treatment and TAVR in patients with prohibitive surgical risk. Therefore, the conclusion and subsequent guidelines recommendations should be based on the population and comparative groups involved. We support palliative care in patients with prohibitive surgical risk in whom TAVI is not feasible.

In patients at high surgical risk, no evidence shows that TAVI is superior to SAVR. The actual evidence is that TAVI is not inferior to SAVR for those at high risk; therefore, guidelines recommendation giving Level of Evidence A should reflect this.

Current evidence shows TAVR to be noninferior to SAVR in patients at high risk; therefore, LACES considers both options to have the same level of recommendation. LACES does not support giving the same recommendation in patients at high and prohibitive risk.

\section{FUNCTIONAL MITRAL REGURGITATION}

After careful consideration of the new 2020 AHA/ACC clinical guidelines for valvular heart disease, we have found several points concerning the recommendations for using transcatheter edge-to-edge mitral valve (MV) repair as a 
treatment in the setting of functional mitral regurgitation (FMR), with which we are totally at odds. If coronary artery bypass grafting is needed, then surgery is indicated as the COR IIA. However, the main disagreement is concerning patients not undergoing coronary artery bypass grafting.

In these new guidelines, transcatheter edge-to-edge MV repair is considered as COR IIA if the case has severe mitral regurgitation (MR) stage $\mathrm{D}$ (regurgitan volume $\geq 60 \mathrm{~mL}$, regurgitant fraction $\geq 50 \%$, effective regurgitant orifice area $\geq 0.4 \mathrm{~cm}^{2}$ ), LV ejection fraction less than $50 \%$, persistent symptoms on optimal guideline-directed medical therapy, with MV anatomy as favorable, LV ejection fraction $20 \%$ to $50 \%$, LV end-diastolic diameter $70 \mathrm{~mm}$ or greater, and pulmonary artery systolic pressure $70 \mathrm{~mm} \mathrm{Hg}$ or less. Recommendations have been based mainly on data from the COAPT trial.

FMR is not a valvular disease, but a final LV condition leading to heart failure. Every single one of the attempts on the MV (surgical and percutaneous) will be just a palliative measure to alleviate the MR. The prognosis remains unaltered, whereas the quality of life or freedom from symptoms can be improved using any treatment directed to mitigate or even eliminate $\mathrm{MR}$, regardless of the approach. MR repair using restrictive annuloplasty is the more reproducible technique for this purpose. Nevertheless, there has been great concern because of the MR recurrence in the short term.

Although it is true that surgical annuloplasty might not be optimal for cases with FMR, it is also true that there are some other surgical options on the MV that can be highly recommended as a definite treatment directed to stop further dilation and remodeling of the LV. MV replacement is another surgical choice. However, one consideration of paramount importance is that all these foregoing facts apply the same regardless of whether the approach is surgical or percutaneous.

Moreover, when comparing the results of the COAPT trial (Abbott Funded) with the MITRA-FR trial (French Ministry of Health and Research National Program Funded), both trials differed considerably in the primary outcome. ${ }^{3,4} \mathrm{~A}$ recently published post hoc analysis of a subgroup of patients from the MITRA-FR trial who met COAPT inclusion criteria, transcatheter edge-to-edge MV repair failed to show superiority over optimal medical therapy. Therefore, longer-term outcomes and additional trials are required.

In light of these details, LACES does not support COR IIA for transcatheter edge-to-edge $M V$ repair in the presence of FMR. The contradicting outcomes between the only 2 trials that have evaluated this technique and the limited 2 to 3 years of data from the COAPT trial do not justify the wide expectations of a COR IIA for the percutaneous approach.

In regard to FMR, all the available long-term information is derived from surgical experience, with a significant long- term follow-up to 14 years or more. From this experience, it has been clear that the most powerful predictor for failure after edge-to-edge MV repair is the lack of an annuloplasty ring. As it stands now in the current version, the transcatheter edge-to-edge MV repair therapy is a ringless technique; therefore, longer-term outcomes are needed before we are able to evaluate the safety of a ringless technique.

In regard to comparison with mitral surgery, the EVEREST II trial included $27 \%$ of patients with FMR. ${ }^{5}$ The primary outcome (freedom from death, surgery in the percutaneous group, MR 3+ or 4+, surgery in the repair group) at 1,2 , and 5 years was significantly worse for the percutaneous edge-to-edge group. There is no other randomized controlled trial that evaluates the long-term outcomes of surgery versus transcatheter edge-to-edge.

Considering the worse long-term outcomes of the transcatheter edge-to-edge (EVEREST II trial) and absence of long-term safety using a ringless technique, LACES does not support a higher level of COR for the percutaneous approach compared with surgery. We believe careful evaluation of surgical risk by a Heart Team should define the best approach.

\section{CONCLUSIONS}

We believe one of the main drivers of big societies such as the AHA, ACC, European Society of Cardiology, and European Association for Cardio-Thoracic Surgery is to thrive to achieve excellence in the treatment of cardiovascular disease worldwide. Scientific societies should acknowledge these to be worldwide leaders in the field. When guidelines start to diverge from a critical assessment of the scientific evidence with unsupported extrapolations, they lose reliability, practice turns arbitrary, and leaders are lost.

This statement has been submitted to several journals to achieve worldwide diffusion of the position of our Association.

\section{References}

1. Otto CM, Nishimura RA, Bonow RO, Carabello BA, Erwin JP III, Gentile F, et al 2020 ACC/AHA Guideline for the Management of Patients With Valvular Heart Disease: Executive Summary: A Report of the American College of Cardiology/American Heart Association Joint Committee on Clinical Practice Guidelines. Circulation. 2020;143:e35-71.

2. Leon MB, Smith CR, Mack MJ, Makkar RR, Svensson LG, Kodali SK, et al; PARTNER 2 Investigators. Transcatheter or surgical aortic-valve replacement in intermediate-risk patients. $N$ Engl J Med. 2016;374:1609-20.

3. Stone GW, Lindenfeld J, Abraham WT, Kar S, Lim DS, Mishell JM, et al; COAPT Investigators. Transcatheter mitral-valve repair in patients with heart failure. $N$ Engl J Med. 2018;379:2307-18.

4. Obadia JF, Messika-Zeitoun D, Leurent G, Iung B, Bonnet G, Piriou N, et al; MITRA-FR Investigators. Percutaneous repair or medical treatment for secondary mitral regurgitation. N Engl J Med. 2018;379:2297-306.

5. Feldman T, Kar S, Elmariah S, Smart SC, Trento A, Siegel RJ, et al; EVEREST II Investigators. Randomized comparison of percutaneous repair and surgery for mitral regurgitation: 5-year results of EVEREST II. J Am Coll Cardiol. 2015; 66:2844-54. 